\title{
Comparison of the intestinal microbiota composition and function in healthy and diseased Yunlong Grouper
}

\author{
Chao Ma* ${ }^{*}$, Chunxiu Chen, Lei Jia, Xiaoxu He and Bo Zhang
}

\begin{abstract}
Maintaining stabilization of the intestinal microbiota is important in preventing bacterial diseases in cultured fish. At present, there have been no reports on the composition and functional analysis of intestinal microbiota in Yunlong Grouper (Epinephelus moarao $\times$ Epinephelus lanceolatus ${ }^{\wedge}$ ). In this study we analyzed and compared the intestinal microbiota composition of healthy and diseased pond-reared fish to discern the functional profile of a healthy status. The richness and diversity of the intestinal microbiota did not differ significantly between diseased and healthy fish, yet the abundance of predominant phyla like the Proteobacteria were upregulated in the diseased Yunlong Grouper. At the genus level, a significant reduction of Cetobacterium was observed in the intestinal tracts of diseased fish, as Pseudomonas became the most dominant bacterium. To compare the intestinal microorganism abundances between the two health groups of fish, we first screened the gut bacteria and discerned 4 phyla and 12 genera to designate a healthy status in Yunlong Grouper. The environmental bacterial community influenced composition of the intestinal microbiota in Yunlong Grouper, and the intestinal microbiota of diseased fish was more susceptible to the influence of the culture water. In addition, the prediction of functional genes by phylogenetic investigation of communities by reconstruction of unobserved states (PICRUSt) indicated that the intestinal microbiota of Yunlong Grouper is related mainly to the terms "metabolism, environmental information processing, genetic information processing, human diseases, and cellular processing; moreover, the functions of the intestinal microbiota differed between the different health states of this fish. The overall results indicate that the occurrence of disease can affect the composition and function of the intestinal microbiota in a cultured fish.
\end{abstract}

Keywords: $16 \mathrm{~S}$ rRNA, Intestinal microbiota, PICRUSt, Yunlong Grouper

\section{Introduction}

The intestinal microbiota comprise a complex and diverse ecosystem attached to the intestinal mucosa and they exist in a dynamic state. Once the balance of the intestinal microbiota is destroyed, the host becomes susceptible to innumerable bacterial diseases. Thus, stability of the intestinal microbiota community is an important factor in preventing bacterial diseases in animals (Round and Mazmanian 2009; Ringø et al. 2003). Likewise in fishes, the intestinal microbiota play an important role in growth and development, nutrient absorption, immunity

*Correspondence: bhsmachao@163.com

Tianjin Bohai Sea Fisheries Research Institute, Tianjin, People's Republic of China and disease resistance (Eddy and Jones 2002; Wu et al. 2013. However, unlike in terrestrial animals, the fish gut is directly coupled to its aquatic environment. The formation of intestinal microflora in cultured fish is not only related to the species of fish, but is also affected by the aquatic environment, food, and other factors(Pérez et al. 2010). Therefore, knowledge of the intestinal and environmental microbiota has great significance for preventing disease and achieving healthy growth in cultured species.

Yunlong Grouper is a hybrid progeny (Epinephelus moara $\times$ E. lanceolatus $\left.{ }^{\star}\right)$ with fast growth, a high survival rate, and wide temperature range. Researchers have reported on their embryonic (Tian et al. 2017a) and post-embryonic development (Wu et al. 2016a, b, c), the 
adaptability of fry to temperature and salinity (Xing et al. 2017), and karyotype analysis (Cheng et al. 2018). However, there has been no research using 16S rRNA gene sequencing to analyze the intestinal microflora of Yunlong Grouper in different health states, nor studies of the correlation between composition of the intestinal microflora and the culture environment.

Phylogenetic investigation of communities by reconstruction of unobserved states (PICRUSt) is a bioinformatics software package used to predict metagenomic functional genes (Morgan et al. 2013). It can predict the metabolic function of bacteria and archaea through $16 \mathrm{~S}$ rRNA gene sequences, and its applications are growing. For example, in one study (Wu et al. 2016a, b, c), PICRUSt predicted the oral flora of smokers and non-smokers, and found that there were significant differences in 83 functional metabolic pathways. Loudon (Loudon et al. 2014) used PICRUSt to identify core bacterial communities on the skin of red-backed salamanders. Functional predictions have revealed that a core bacterial community is closely linked to immune regulation and plays an important role in maintaining the basic functions of the body.

At present, the potential relationship between changes in the composition and the function of the intestinal microflora in this species remain unclear. Therefore, this study used healthy and diseased Yunlong Grouper and samples of their culture water and feed as research objects. 16S rRNA sequencing technology was used to analyze the composition of the intestinal microbiota in different health states of the fish. PICRUSt was applied to predict gene function, evaluate changes in the intestinal microbiota function, and study the correlation with the culture environment. Furthermore, from the perspective of micro-ecology, this investigation of the influence of disease on the composition and function of the intestinal microbiota of Yunlong Grouper will provide a scientific basis and reference for disease prevention and the healthy breeding of Yunlong Grouper.

\section{Materials and methods \\ Sample collection}

Yunlong Grouper and their feed and culture-water were sampled at the Tianjin Xingsheng Sea and Freshwater Aquaculture Co., Ltd. All sampled fish were of the same age ( 6 months old) and had been fed the same feed and reared in a similar environment ( $\mathrm{n}=3$ per group). Two experimental groups were acquired from same recirculating aquaculture systems: healthy fish ( $\mathrm{H}$ group) and diseased fish (D group); the latter were taken from a culture pond specifically maintained for producing diseased fish, and all the fish had obvious signs of disease by visual observation. The healthy samples were randomly removed from a culture pond and visually observed for signs of disease before being dissected. Mean $( \pm S D)$ of body length (BL) was $38.33 \pm 3.79 \mathrm{~cm}$ for the $\mathrm{H}$ group vs. $30.67 \pm 1.53 \mathrm{~cm}$ for the $\mathrm{D}$ group ( $t$ test, $P=0.12$ ); body weight (BW) was $684.13 \pm 124.24 \mathrm{~g}$ for the $\mathrm{H}$ group vs. $374.23 \pm 71.02 \mathrm{~g}$ for the $\mathrm{D}$ group $(t$-test, $P=0.11)$. There were three replicates for each of the two health-status groups. Sterile water-collection bottles (2 L) were used to take culture water from four locations in each of the two $20-\mathrm{m}^{3}$ culture ponds. Feed samples were collected using sterile bags. All samples were immediately transported to the laboratory.

\section{DNA extraction and sequencing of 16S rRNA genes}

Prior to autopsy, the skin of sampled fish was disinfected with $70 \%$ ethanol. The gut was removed and placed into a $1.5-\mathrm{mL}$ centrifuge tube, and immediately stored at $-80{ }^{\circ} \mathrm{C}$ until DNA extraction. DNA was extracted from the intestinal contents using a QIAamp DNA Stool Mini Kit (Qiagen, Germany), according to the manufacturer's instructions. The 2-L water samples were sequentially filtered through $0.22-\mu \mathrm{m}$ filter paper (Jin Teng, China) to collect as many bacterioplankton as possible; DNA was extracted from these organisms using the E.Z.N.A. ${ }^{\circledR}$ Water DNA Kit (Omega Bio-Tek, Norcross, GA, USA) according to the manufacturer's instructions. The V4V5 variable region of $16 \mathrm{~S}$ rRNA genes of gut microbiota were amplified by polymerase chain reaction (PCR) using the primers 515F [ $5^{\prime}$-GTGCCAGCMGCCGCGGTAA-3' (Caporaso et al. 2011)] and 926R [5'-CCGTCAATTYYT TTRAGTTT-3' (Liu et al. 2007)]. Next, the total bacterial DNA of each sample, extracted by the above method, was constructed by a two-step PCR amplification method. The first PCR reaction reaction conditions were $94^{\circ} \mathrm{C}$ for $2 \mathrm{~min}$; and $94{ }^{\circ} \mathrm{C}$ for $30 \mathrm{~s}, 56^{\circ} \mathrm{C}$ for $30 \mathrm{~s}, 72{ }^{\circ} \mathrm{C}$ for $30 \mathrm{~s}$, $72{ }^{\circ} \mathrm{C}$ for $5 \mathrm{~min}, 25$ cycles. The second PCR conditions were $94{ }^{\circ} \mathrm{C}$ for $2 \mathrm{~min}$; and $94{ }^{\circ} \mathrm{C}$ for $30 \mathrm{~s}, 56{ }^{\circ} \mathrm{C}$ for $30 \mathrm{~s}$, $72{ }^{\circ} \mathrm{C}$ for $30 \mathrm{~s}, 72{ }^{\circ} \mathrm{C}$ for $5 \mathrm{~min}, 10^{\circ} \mathrm{C}$ heat preservation, for 8 cycles.

All PCR products were recovered from the gel with an AxyPrep DNA Gel Recovery Kit (Axygen, USA) and then quantified using an FTC- $3000^{\mathrm{TM}}$ Real-Time PCR system. The amplicons were sequenced with Illumina MiSeq $2 \times 300$ bp (Illumina Inc., San Diego, CA, USA). Sequencing was carried out at TinyGene Bio-Tech Co., Ltd (Shanghai, China).

\section{Statistical methods}

All sequences were clustered into operational taxonomic units (OTUs) by de novo OTU picking at the $97 \%$ similarity level, performed with the software Mothur (Schloss et al. 2009). The Ribosomal Database Project (RDP) Classifier (Version 2.2; https://sourceforge.net/proje 
cts/rdp-classifier) and the Greengenes Database (http:// greengenes.lbl.gov) were used for taxonomic assignments. Rarefaction curves and Alpha diversity indices (i.e. ACE, Chao1, Shannon and Simpson) were generated with Mothur (Schloss et al. 2009). Significant differences of alpha diversity index between groups were calculated with a Kruskal-Wilcox test. Principal coordinates analysis (PCoA) with the weighted UniFrac distance was conducted based on the phylogenetic information (Lozupone et al. 2006). For bar plots and heatmap analysis, the PCoA was calculated and plotted in R (Version 3.4.1), and the hcluster analysis was plotted in QIIME (Version 1.9.1). Statistical differences were analyzed with SPSS 13.0 (SPSS Inc., Chicago, IL, USA) and Metastats (http:// metastats.cbcb.umd.edu) software. A $P$-value of less than 0.05 was considered significant.

Characterization of the healthy and diseased Yunlong Grouper in each treatment was performed using linear discriminant analysis (LDA) effect size (LEfSe) method (http://huttenhower.sph.harvard.edu/lefse) for biomarker discovery. The LEfSe method uses the Kruskal-Wallis rank sum test to detect features with significantly different abundances between the assigned taxa and performs the LDA to estimate the effect size of each feature with the normalized relative abundance matrix.

\section{Functions prediction}

PICRUSt was employed to predict the functional profiles of microbial communities (Morgan et al. 2013). After normalized of the $16 \mathrm{~S}$ rRNA copy numbers, the microbiota functions were predicted with reference to the Kyoto Encyclopedia of Genes and Genomes (KEGG) Orthology (KO) database (Kanehisa et al. 2012).

\section{Results}

\section{6s rRNA sequencing data and alpha-diversity analysis}

By sequencing the V4-V5 region of the 16s rRNA gene from the samples, the high-throughput sequencing data

Table 1 The sequencing depth of each sample

\begin{tabular}{lllc}
\hline Sample name & Effective tags & Clean data & $\begin{array}{l}\text { Numbers } \\
\text { of OTUs }\end{array}$ \\
\hline HW & 35,768 & 22,173 & 275 \\
DW & 33,639 & 21,091 & 280 \\
AF & 33,890 & 25,893 & 69 \\
H1 & 29,297 & 27,344 & 155 \\
H2 & 36,262 & 34,280 & 108 \\
H3 & 34,751 & 32,727 & 50 \\
D1 & 37,177 & 28,683 & 178 \\
D2 & 37,025 & 31,829 & 198 \\
D3 & 34,530 & 27,884 & 145 \\
\hline
\end{tabular}

in each sample can be statistically analyzed. The effective tags of each sample were between 29,297 and 37,177, and the optimized clean data were between 21,091 and 34,280 . A total of 521 OTUs were obtained, which identified to 18 phyla. The samples of healthy fish aquaculture water (HW), diseased fish aquaculture water (DW), feed (AF), healthy fish intestinal microbiota $(\mathrm{H} 1, \mathrm{H} 2, \mathrm{H} 3)$, and diseased fish intestinal microbiota (D1, D2, D3), respectively, included $275,280,69,155,108,50,178$, 198 and 145 OTUs (Table 1). The number of OTUs were $104.33 \pm 52.60$ for healthy fish, and $173.67 \pm 26.76$ for diseased fish; the difference between the two groups was not significant $(P>0.05)$.

The rarefaction curve was drawn with randomly selected sequence numbers and the number of OTUs they can represent (Fig. 1). The results showed that the curve of each sample tended to flatten with higher sequence numbers, indicating that the amount of sequencing data was reasonable. The alpha diversity index of the two groups is shown in Table 2. The

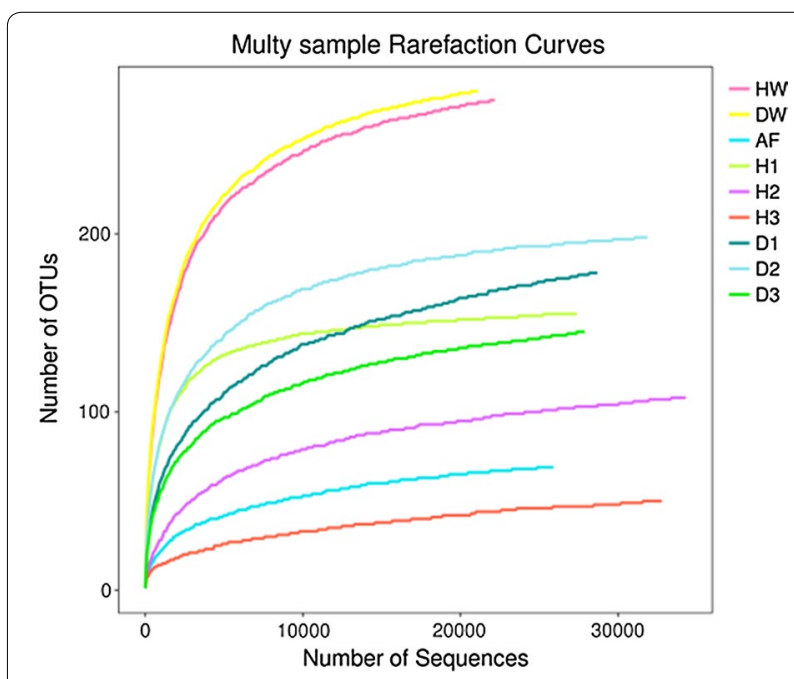

Fig. 1 Rarefaction curve. $\mathrm{H1}-\mathrm{H} 3$ represent the intestinal microbiota of three healthy fish; D1-D3 represent the intestinal microbiota of three diseased fish; HF healthy fish aquaculture water, DW diseased fish aquaculture water, $A F$ feed

Table 2 Analysis of alpha diversity indices for the healthy and the diseased groups of fish (mean \pm SD)

\begin{tabular}{lccl}
\hline Index & Healthy group & Diseased group & $\begin{array}{l}\text { Wilcox- } \\
\text { test } \\
\text { P-value }\end{array}$ \\
\hline Chao1 & $118.86 \pm 27.74$ & $194.45 \pm 15.75$ & 0.081 \\
ACE & $119.53 \pm 27.20$ & $195.69 \pm 15.88$ & 0.081 \\
Shannon & $1.80 \pm 0.60$ & $2.56 \pm 0.34$ & 0.383 \\
Simpson & $0.34 \pm 0.11$ & $0.20 \pm 0.06$ & 0.383 \\
\hline
\end{tabular}


Chao1 and ACE indices reflected the species richness of the intestinal microbiota in the samples: these were $118.86 \pm 27.74$ and $119.53 \pm 27.20$, respectively, for the healthy fish group, and $194.45 \pm 15.75$ and $195.69 \pm 15.88$ for the diseased fish group. The Shannon and Simpson indices reflected the diversity of the intestinal microbiota in the samples: these were $1.80 \pm 0.60$ and $0.34 \pm 0.11$, respectively, for the healthy fish group, and $2.56 \pm 0.34$ and $0.20 \pm 0.06$ for the diseased fish group. The Wilcoxtest was used to analyze differences in the alpha diversity index between the healthy and the diseased fish groups, and the results showed no significance $(P>0.05)$.

\section{Composition of the intestinal microbiota}

To estimate the abundance of taxa, the OTUs identified a total of 18 phyla in the fish intestinal microbiota, feed, and culture-water samples. The microbiota composition of the healthy fish aquaculture water was similar to that of the diseased fish aquaculture water. Both showed Proteobacteria, Bacteroidetes, and Actinobacteria as the dominant phyla, and total relative abundance was $>80 \%$. The microbiota composition of the feed was relatively simple, with Proteobacteria as the dominant phyla, with a relative abundance of $94 \%$ (Fig. 2a). The three mostdominant intestinal microbiota taxa in healthy fish were Proteobacteria (54.02\%), Fusobacteria (23.30\%), and Firmicutes (20.47\%), while that of diseased fish comprised Proteobacteria (56.21\%), Actinobacteria (16.00\%), and Cyanobacteria (12.61\%) (Fig. 2b).

After further analysis of the sample data, each sample of fish intestinal microbiota was screened for the dominant genera (relative abundance $>0.5 \%$ ), and the microbiota composition and proportions of each group were analyzed at the genus level (Fig. 2c). The most-dominant genera in healthy fish intestinal microbiota were Cetobacterium and Pseudomonas, with a relative abundance of $23 \%$. The dominant genera in the diseased fish group was Pseudomonas, with a relative abundance of $32 \%$, whereas the relative abundance of Cetobacterium was only $0.5 \%$.

\section{Sensitive microbial screening}

LEfSe analyses (LDA values are shown in Fig. 3) were performed to determine whether there were significant differences in taxon abundance between the two fish groups (Fig. 4). For classification of phylum, the biomarkers emerging for the healthy fish group indicated
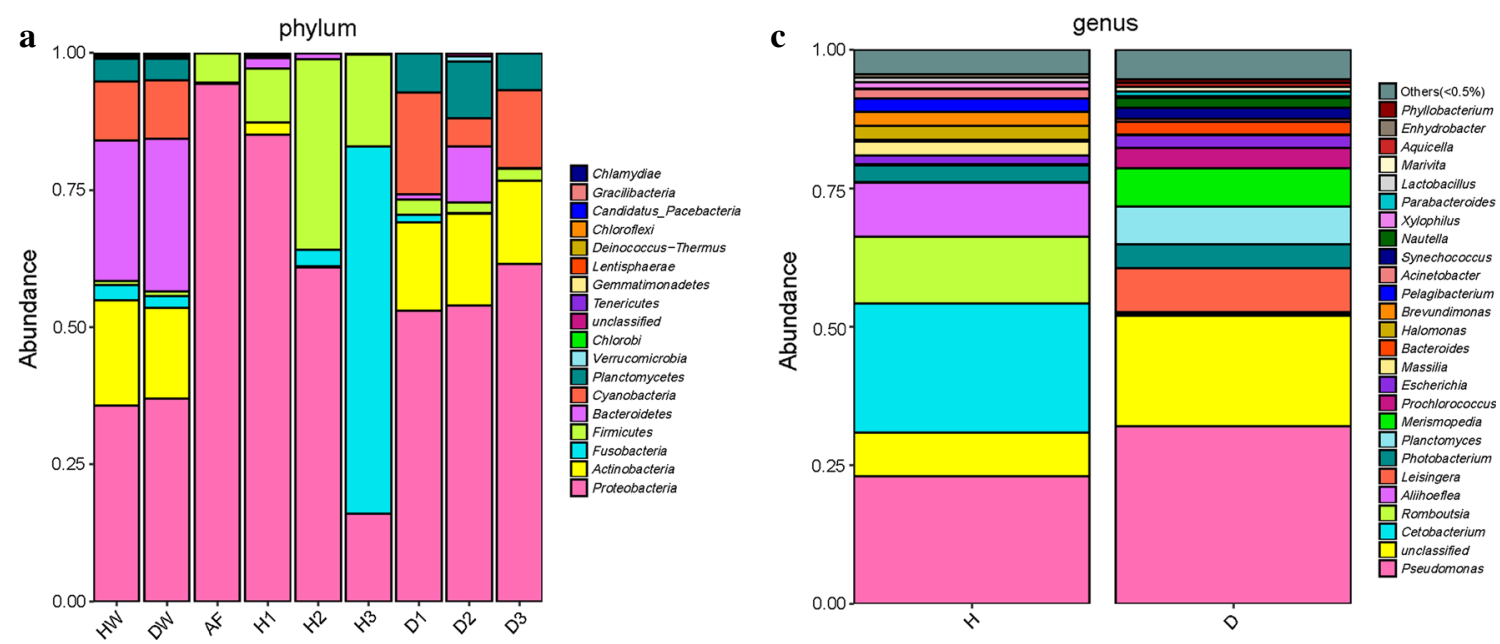

b

$\mathrm{H}$

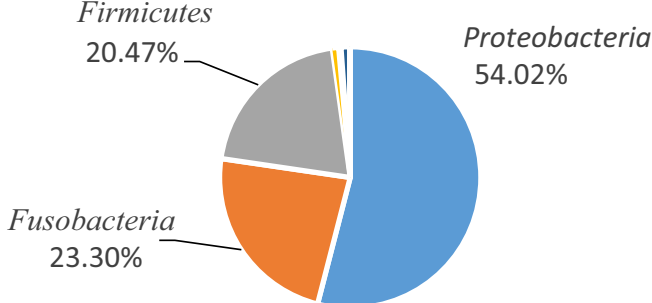

D

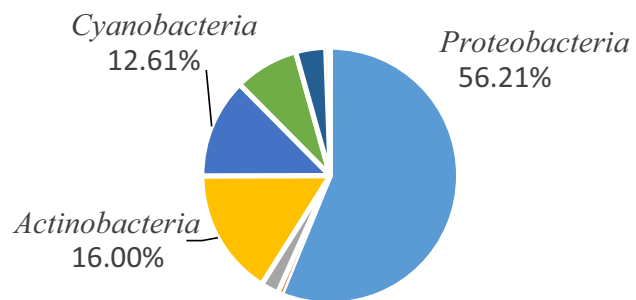

Fig. 2 a Relative abundance of each sample at the taxonomic level of phylum. $\mathbf{b}$ Comparison of the relative abundances of the dominant intestinal microbiota phyla between the healthy and diseased Yunlong Grouper. c Relative abundance of the dominant genera (mean relative abundance $>0.5 \%$ ) in the intestinal microbiota between the healthy and the diseased Yunlong Grouper 


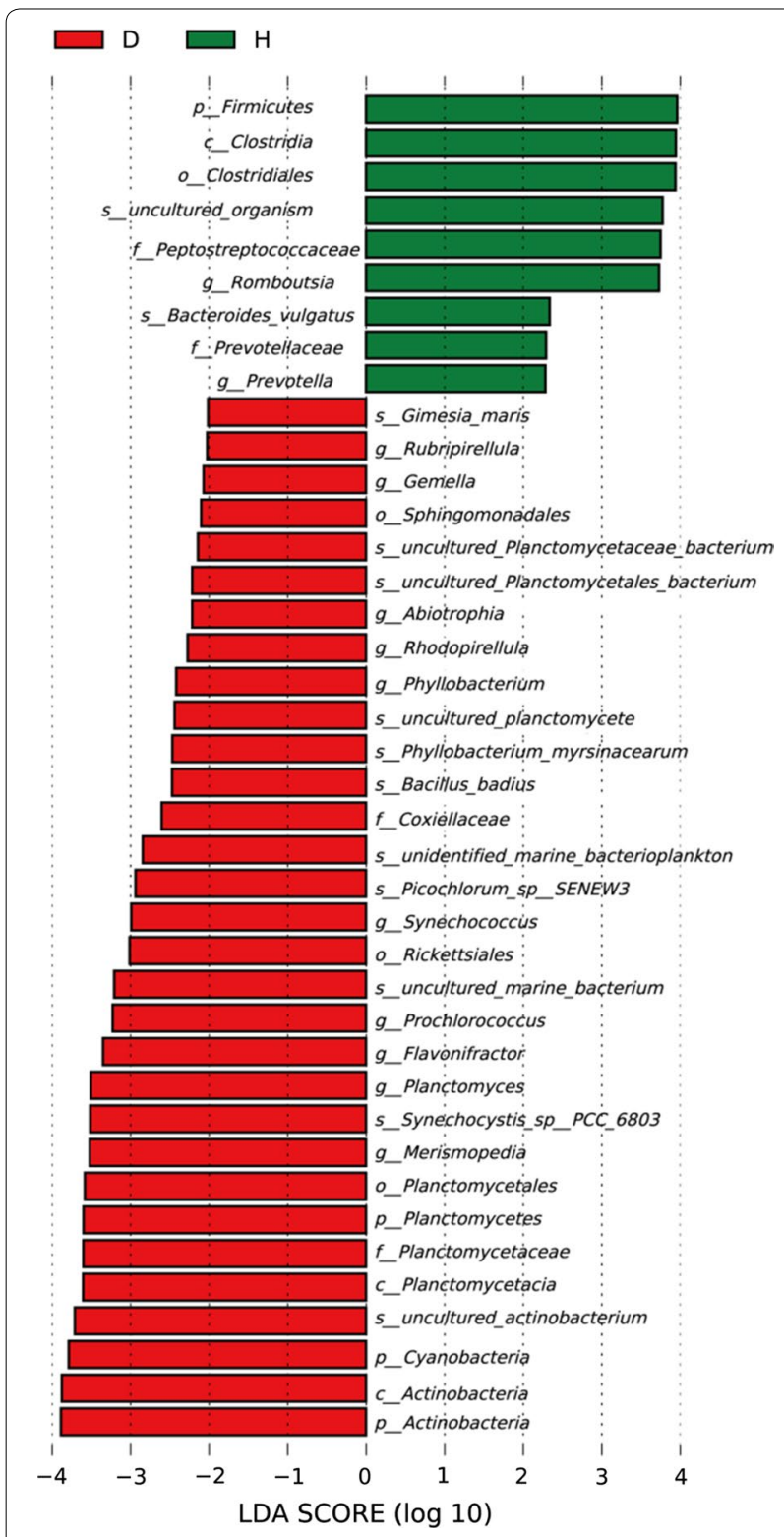

Fig. 3 LDA scores of the intestinal microbiota in the healthy and the diseased Yunlong Grouper. A significance alpha of 0.05 and an effect size threshold of 2 were used for all biomarkers evaluated

Firmicutes; Actinobacteria and Planctomycetes were biomarkers for the diseased group; and Actinobacteria had the highest LDA score.

\section{Beta diversity analysis}

PCoA with weighted UniFrac distance and heatmap analysis were used to compare the overall composition of the microbiota between the fish intestine and the environment (Figs. 5, 6). The plot of PCoA scores shows that the environmental samples and the fish intestinal microbiota samples are separated along PC1 and accounted for $39.73 \%$ of the total variation, except for $\mathrm{H} 1$ and D2, which may be caused by individual differences. All the diseased fish intestinal samples and culture-water samples were above PC2. All the healthy fish intestinal tract samples and the feed sample were below $\mathrm{PC} 2$, and account for $34.01 \%$ of the total variation. Moreover, the composition of each intestinal microbiota sample in the healthy fish group was unique. Overall, the two PCOA axes explained more than $73 \%$ of the total variation among the different communities. The heatmap of bacterial microbiota at the genus level reflects the similarities and differences in the sample microbiota composition through color gradients and degree of similarity. The results showed that the microbiota composition was similar in the healthy and diseased fish groups.

\section{Functional analysis}

To analyze the functional changes of the intestinal microflora as a consequence of disease, metagenomes potential between the two groups were predicted by PICRUSt. The results revealed that high abundance of bacterial metagenome in the two groups was mainly associated with "metabolism," "environmental information processing," "genetic information processing," "human diseases" and "cellular processing" in KEGG level 1 (Fig. 7a). For "metabolism," the largest difference between the two groups was found for the level-2 terms "metabolism of Terpenoids and Polyketides" and "biosynthesis of other secondary metabolites." Within "human diseases," the abundance of metabolic diseases was significantly higher in the diseased group of fish than in the healthy group (Fig. 7b). In KEGG level 3, a total of 42 KEGG pathways changed significantly between the two groups by Metastats (data not shown). In the parent transport and catabolism and carbohydrate metabolism, significantly different abundances of carbohydrate metabolism were observed in the diseased group (Fig. 7c).

\section{Discussion}

Because of its high data flux, sequencing depth and accuracy, second-generation high-throughput sequencing technology has been widely used in aquaculture, especially in the study of fish intestinal microbiota. Recent studies have found that changes in intestinal microbial diversity and imbalances of the intestinal microbiota have different effects on host fishes (Gómez and Balcázar 2010; Willing et al. 2011). Zhang et al. (2014) found that tongue sole Cynoglossus semilaevis with ascites displayed a significantly altered composition of the dominant intestinal microbiota. Other studies have shown that intestinal microbes can help the host to complete in regard to a variety of physiological and biochemical functions, 


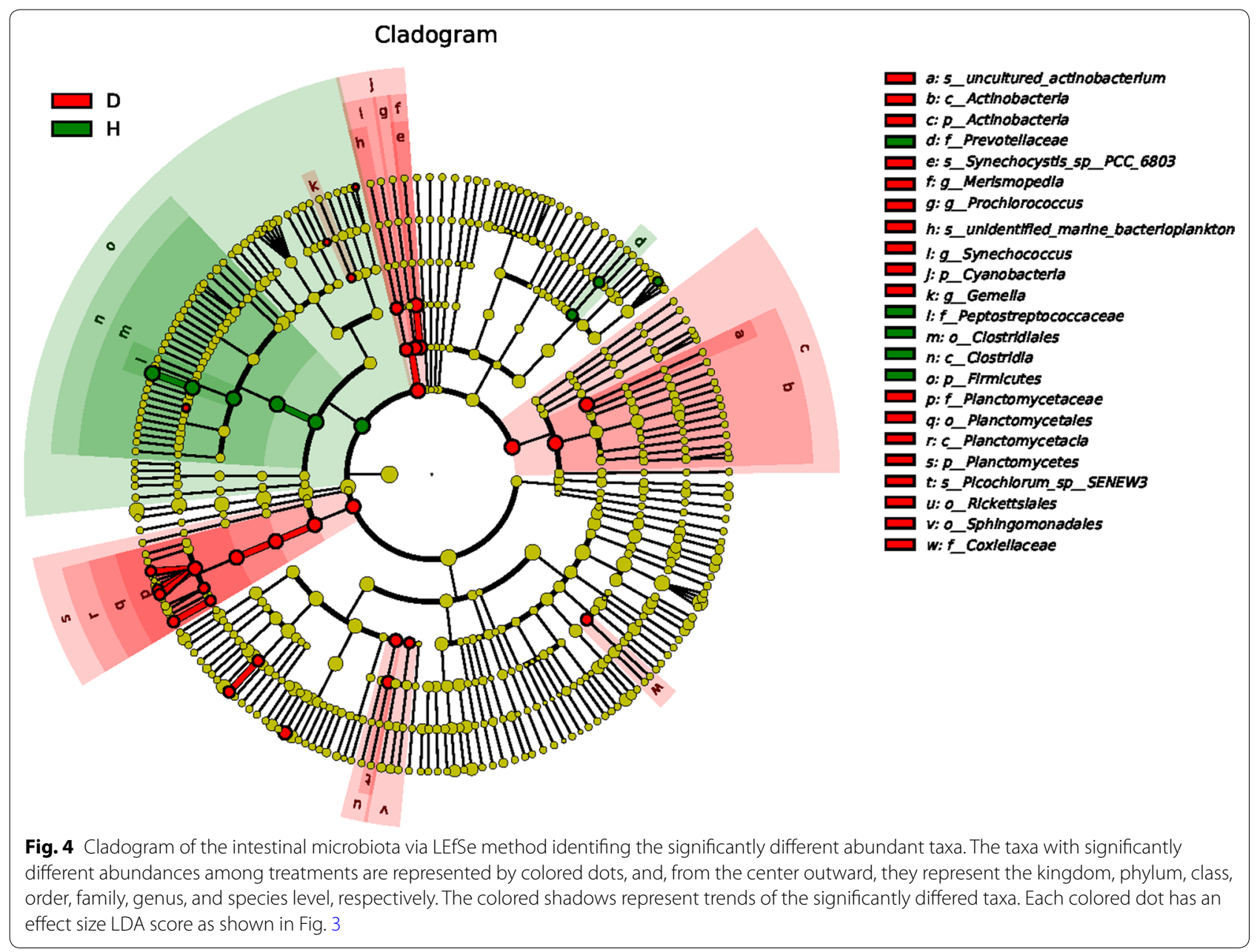

including promoting the digestion and absorption of food, regulating the host immune system, and resisting pathogenic bacteria (Karasov et al. 2011; Forsythe and Bienenstock 2010).

To better understand the relationship between intestinal microbiota composition and fish health condition, the intestinal microbiota of Yunlong Grouper was examined. We found no significant difference between the taxonomic richness and diversity of the intestinal microbiota in diseased fish and healthy fish. The dominant phyla in the intestinal microbiota of healthy fish were Proteobacteria (54.02\%), Fusobacteria (23.30\%), and Firmicutes (20.47\%); while in the intestinal microbiota of diseased fish they were Proteobacteria (56.21\%), Actinobacteria (16.00\%), and Cyanobacteria (12.61\%). The composition of the intestinal microbiota of diseased fish had somewhat changed from the structure present in a healthy state. Proteobacteria was the most dominant phyla in the microbiota community of Yunlong Grouper. This finding is consistent with previous studies of other species of marine and freshwater fishes, including grass carp, yellow catfish, coho salmon, and rainbow trout (Wu et al. 2012, 2016a, b, c; Kim et al. 2007; Romero and Navarrete 2006). Although the occurrence of disease affected the relative abundance of Proteobacteria in Yunlong Grouper intestinal microbiota, it did not change its position as the most-dominant phyla in the intestinal tract. The composition of the intestinal microbiota were analyzed and compared in the healthy and diseased fish groups at the genus level; the dominant genus in the healthy group was Cetobacterium, with a relative abundance of $23 \%$, while in the unhealthy group it was Pseudomonas, with a relative abundance of $32 \%$, with Cetobacterium amounting to only $0.5 \%$. Previous research found that Cetobacterium, isolated from the intestines of five different species of freshwater fish, conveyed the effects of fermented polypeptide carbohydrate and could produce vitamin $B_{12}$, suggesting that Cetobacterium plays an important role in processes of digestion and nutrition in fishes (Tsuchiya et al. 2008). Furthermore, vitamin B12 can promote protein biosynthesis, acting as a growth factor in many animals, and a lack of which will generally affect growth 


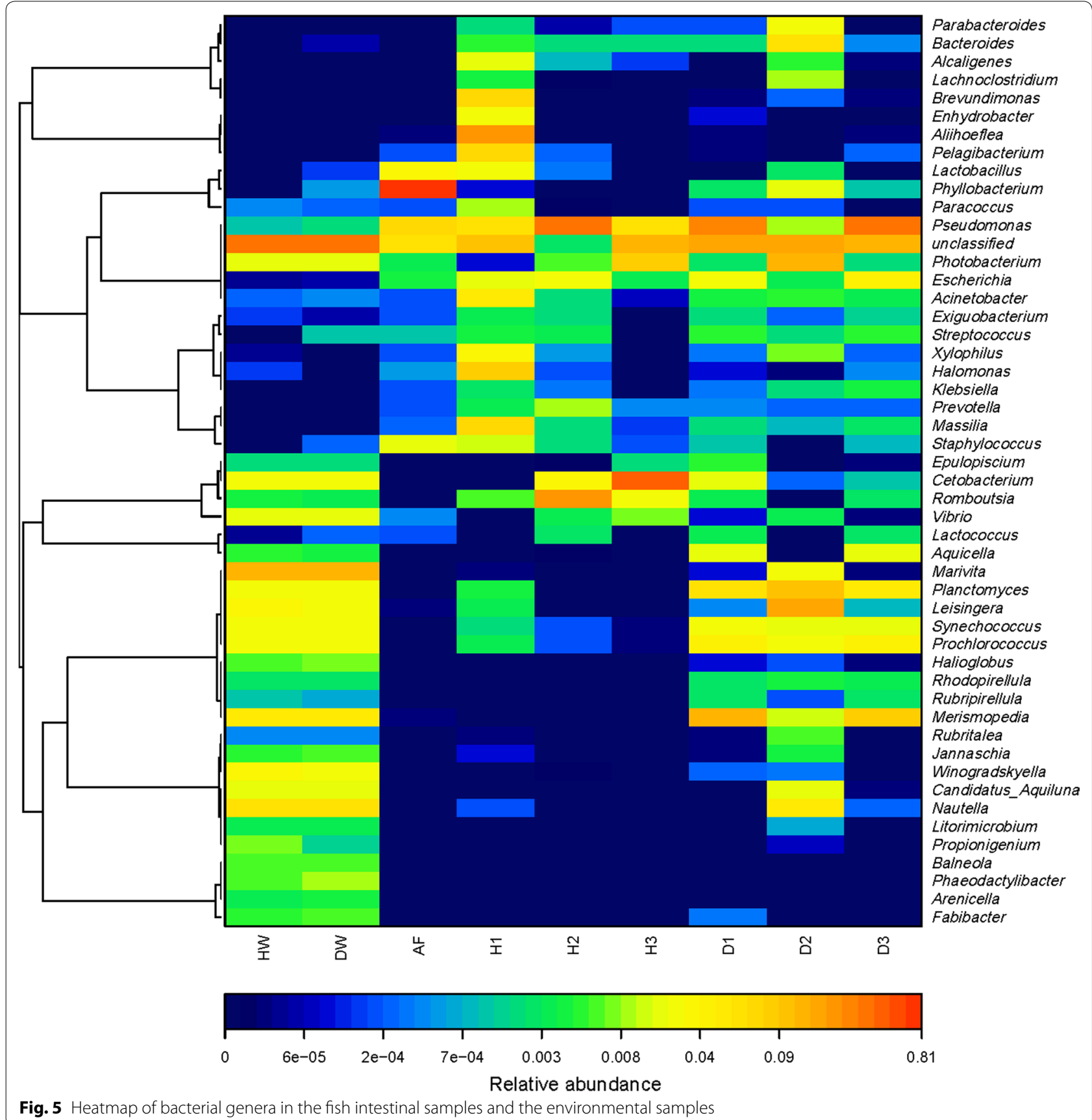

and development (StroInsky and SchneIder 1987; Hunik 2002). Likewise, many studies have shown that vitamin $\mathrm{B}_{12}$ helps to maintain normal growth in fishes (Arai et al. 1972; Ikeda et al. 1988). In the current study, a comparison of the indices of body weight and body length for the two groups of Yunlong Grouper showed no significant differences between the diseased group and the healthy group. However, the growth of the diseased fish was relatively slow, with the average body weight only $54.7 \%$ that of the healthy fish, and the average body length only $80 \%$ that of the healthy fish. Pseudomonas, a common bacterial genus in seawater, is speciose and has great interspecies differences. The pathogenicity of Pseudomonas depends mainly on the physiological state of the fish body and the physical and chemical conditions of the water. Studies have shown that Pseudomonas infections occur in both warm- and cold-water marine fishes as well as in freshwater fishes, for example, the gill-rot disease of 


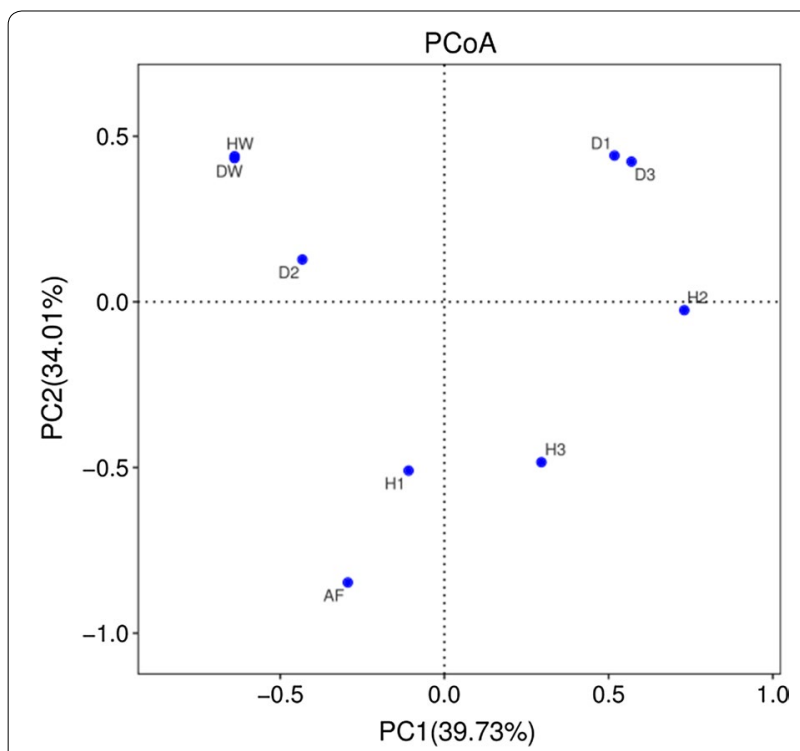

Fig. 6 Principal coordinates analysis (PCOA) plots of beta diversity based on a weighted UniFrac distance metric

Anguilla anguilla (Fan 2001) and the Debonding disease of Fugu obscurus (Li et al. 2001). Thus, it can be inferred that Cetobacterium has the function of promoting food digestion and absorption, and general growth and development processes in Yunlong Grouper. Consequently, these bacteria can be used as probiotics in the breeding of Yunlong Grouper. However, the intestinal microbiota composition becomes disordered in diseased fish, allowing a large number of opportunistic pathogens (especially Pseudomonas) to proliferate; this situation may inhibit the growth of beneficial bacteria and lead to slow growth of the diseased fish. Nonetheless, the effects of Pseudomonas on the intestinal microbiota of fishes merits further study.

In this study, LEfSe software was used to analyze differences in the relative abundance of intestinal microbiota in different health states of Yunlong Grouper, and to estimate the effects of the differences. Bacteria from 4 phyla and 12 genera were screened. Among them, Actinobacteria had the highest LDA score in the intestinal microbiota of diseased fish, while in the healthy fish the score was highest for Firmicutes. Actinobacteria have the ability to biosynthesize secondary metabolites as antibiotics against invasive pathogens (Penn et al. 2009). Studies of humans have shown that patients with inflammatory bowel disease displayed a reduction in the relative abundance of Firmicutes and an increase of Proteobacteria (Blumberg and Powrie 2012; Reveco et al. 2014). That findings is consistent with our results. It is speculated that the intestinal tracts of the diseased groupers were inflamed to a certain extent, yet further study is merited on these changes in the proportions of Actinobacteria and Firmicutes in the intestinal microbiota, and their effects on the health status of fish.

Because fish live in a complex aquatic environment, so the composition of the intestinal microbiota is more susceptible to the environmental influences of the water (Ganguly and Prasad 2012). Though feed is an important factor that will affect the structure of fish intestinal microbiota (Lu et al. 2011; Dhanasiri et al. 2011; Hao et al. 2015), fish may also ingest a large number of microorganisms in the water column and sediments as well as other food items in their environment (Mandal et al. 2009). In this study, the intestinal microbiota of Yunlong Grouper was influenced by the environmental bacterial community. However, the intestinal microbiota of different individual diseased fish was relatively similar, and was highly similar to the community present in the culture water, indicating the relative susceptibility of the intestinal microbiota of diseased fish to the influence of the culture water. In contrast, every healthy fish exhibited a fairly unique composition of intestinal microbiota, which is similar to the conclusion reached by WANG (Wang et al. 2018) in a study of the intestinal microbial flora of healthy and unhealthy Atlantic salmon reared in a circular aquaculture system. In addition, we found that the feed had a greater impact on the healthy fish than on the diseased fish. Combined with the results of the intestinal microbiota and function prediction, it may be that the feeding ability of the diseased Yunlong grouper had been affected and caused them to consume less.

Previous high-throughput sequencing analysis of fish intestinal microbiota focused mainly on the microbial community structure (alpha diversity and beta diversity), composition, distribution characteristics and influencing factors, and less research has been carried out on microbial community function. More recently, PICRUSt has been widely employed for function prediction of fish intestinal microbiota (Bairagi et al. 2002; Yang et al. 2018; Lyons et al. 2017). We used PICRUSt to obtain the functional composition and determine differences in the intestinal microbiota in different health states of Yunlong Grouper. The PICRUSt analysis revealed functional richness in the intestinal microbiota of Yunlong Grouper, with many microbial functional genes related to "metabolism," "environment information processing," "genetic information processing," "human diseases" and "cellular processing." It was inferred that the intestinal microbiota of Yunlong Grouper played a role in digestion, the immune system, and adaptation to environmental changes. PICRUSt cluster analysis showed significant differences in intestinal function between the healthy and diseased fish groups. The terminal intestinal microflora of rainbow trout from different sources was found to have more sequences related to the metabolism of 


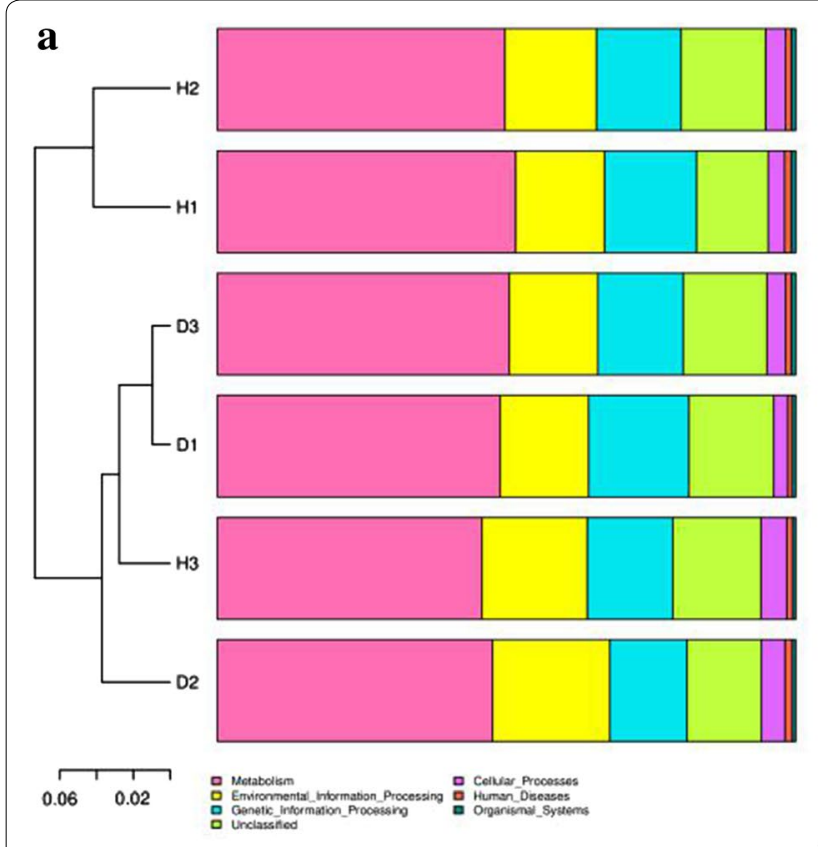

b
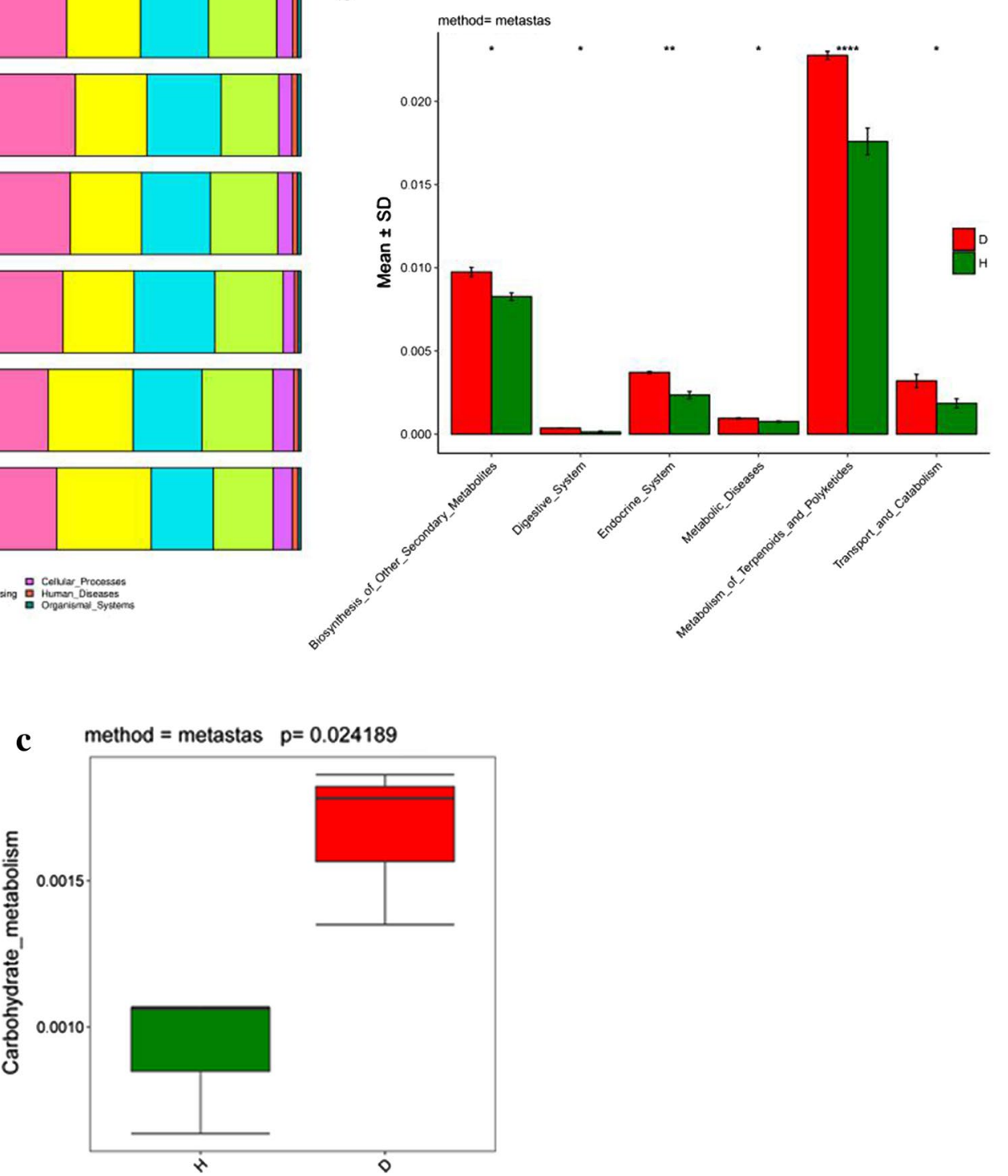

Fig. 7 a The functional composition of the intestinal microbiota in Yunlong Grouper and cluster analysis. $\mathbf{b}$ In the level-2 term, significant changes are evident in intestinal microbiota KEGG pathways between healthy and diseased Yunlong Grouper, determined by the software Metastats. $\mathbf{c}$ Box-plots showed the significant predictive functions (carbohydrate metabolism) of the intestinal microbiota in both the healthy and diseased Yunlong Grouper

sugar, protein and amino acid; and it was inferred that the intestinal microflora exerts some effect on host nutritional metabolism (Lyons et al. 2017). At present, cost-efficient feeds with high carbohydrate and high fat contents are widely used in aquaculture (Xie et al. 2017). Carbohydrates need to be digested to monosaccharides prior to absorption in the small intestine (Kanehisa et al. 2017). In this study, carbohydrate metabolism were significantly greater in the intestines of diseased fish than in healthy fish. Although the abundance of genes involved in the physiological processes of carbohydrate digestion and absorption increased significantly with the occurrence of disease, the energy requirement of the immune system also increased, thus likely reducing the energy that was allocated to growth. The occurrence of disease in the Yunlong Grouper was accompanied by changes in the intestinal flora structure, which in turn results in changes in intestinal function, thereby affecting the functions related to digestion, absorption and energy metabolism. This will lead to abnormal nutrient absorption 
and a poor immune response in the fish, and slower growth for diseased fish as compared with healthy fish.

In this study we found no significant differences in the taxonomic richness and diversity of intestinal microbiota between healthy and diseased Yunlong Grouper, though the composition of the intestinal microbiota had certainly changed. In the intestinal microbiota of healthy fish, probiotics known to promote the digestion and absorption of food and also promote general growth and development are enriched. Pathogenic bacteria may occupy a dominant position in the intestinal microbiota of diseased fish, thus altering the function of the intestinal microbiota, in turn influencing the related functions of digestion, absorption and energy metabolism; which is more likely to lead to appearing the intestinal tract inflammation of Yunlong Grouper, and appearing the abnormal phenomena of growth of diseased fish slower than the growth of healthy fish. These results provide some basis for early detection of infectious bacterial diseases in this newly cultivated hybrid fish. Future research should increase the number of samples to eliminate individual differences, adding accuracy and reliability to the data.

\section{Acknowledgements}

This study was supported by Agriculture Research System of China (CARS47-Z1), Project for Tianjin Fisheries InnovativeTeam (ITT-FRS2018045), Tianjin Agricultural Science and Technology Achievement Conversion and Extension Project (201703050).We greatly appreciate to TinyGene Technologies Co., Ltd., Shanghai, China for the determination of bacterial community structure using high-throughput sequencing using high-throughput sequencing.

\section{Authors' contributions}

CM, CC and $\amalg$ conceived and designed the study. CM., CC, XH and BZ provided the mutants. CM wrote the paper. CC, LJ, XH and BZ reviewed and edited the manuscript. All authors read and approved the final manuscript.

\section{Funding}

Not applicable.

\section{Availability of data and materials}

All sequences analyzed in this study can be accessed in the SRA database under the accession number SRP 220406 (https://www.ncbi.nlm.nih.gov/Trace s/study/?acc=SRP220406). The data set supporting the conclusions of this article is included within the article. Data and materials can also be requested from the corresponding author.

\section{Ethics approval and consent to participate}

The study was approved by the Academic Ethics Committee of Tianjin Bohai Sea Fisherise Research Institute.

\section{Consent for publication}

Not applicable.

\section{Competing interests}

The authors declare that they have no competing interests.

Received: 2 August 2019 Accepted: 9 November 2019

Published online: 21 November 2019

\section{References}

Arai S, Nose T, Hashimoto Y (1972) Qualitative requirements of young eels, Anguilla Japonica, for water-soluble vitamins and their symptoms. Bull Freshwater Res Lab Tokyo. 22:69-83

Bairagi A, Ghosh KS, Sen SK, Ray AK (2002) Enzyme producing bacterial flora isolated from fish digestive tracts. Aquacult Int 10(2):109-121

Blumberg R, Powrie F (2012) Microbiota, disease, and back to health: a metastable journey. Sci Transl Med 4(137):137

Caporaso JG, Lauber CL, Walters WA, Berg-Lyons D, Lozupone CA, Turnbaugh PJ, Fierer N, Knight R (2011) Global patterns of 16S rRNA diversity at a depth of millions of sequences per sample. Proc Natl Acad Sci USA 108:4516-4522

Cheng ML, Tian YS, Wu YP, Li ZT, Zhang JJ, Wang LN, Li WS, Ma WH, Wang XM, Zhai JM, Li B (2018) Chromosome Karyotype Analysis of Hybrids of Epinephelus moaraㅇ $\times$ Epinephelus lanceolatuso. Progr Fish Sci 2:267-275

Dhanasiri AKS, Brunvold L, Brinchmann MF, Korsnes K, Bergh Ø, Kiron V (2011) Changes in the Intestinal Microbiota of Wild Atlantic cod Gadus morhua L., Upon Captive Rearing. Microbial Ecol 61(1):20-30

Eddy SD, Jones SH (2002) Microbiology of summer flounder Paralichthys dentatus fingerling production at a marine fish hatchery. Aquaculture 211(1):9-28

Fan HP (2001) Study on the gill-rot disease of Anguilla anguilla infected by Pseudomonas. J Fish China 2:147-150

Forsythe P, Bienenstock J (2010) Immunomodulation by commensal and probiotic bacteria. Immunol Invest 39(4-5):429-448

Ganguly S, Prasad A (2012) Microflora in fish digestive tract plays significant role in digestion and metabolism. Rev Fish Biol Fisheries 22(1):11-16

Gómez GD, Balcázar JL (2010) A review on the interactions between gut microbiota and innate immunity of fish. FEMS Immunol Med Microbiol 52(2):145-154

Hao YT, Wu SG, Wang GT, Zou H, Li WX (2015) Study on the adaptation changes of intestinal microbiota in Ctenopharyngodon idellus fed on different diets. Freshw Fish 45(3):46-51

Hunik JH (2002) Process for the production of vitam in $B_{12}$. US Patent 6. Dec., 492,141B1

Ikeda S, Ishibashi Y, Murata O, Nasu T, Harada T (1988) Qualitative requirements of the Japanese parrot fish for watersoluble vitamins. Nippon Suisan Gakkaishi 54:2029-2035

Kanehisa M, Goto S, Sato Y, Furumichi M, Tanabe M (2012) Kegg for integration and interpretation of large-scale molecular data sets. Nucleic Acids Res 40(D1):D109-D114

Kanehisa M, Furumichi M, Tanabe M, Sato Y, Morishima K (2017) KEGG: new perspectives on genomes, pathways, diseases and drugs. Nucleic Acids Res 45(D1):D353-D361

Karasov WH, Rio-del CM, Caviedes-Vidal E (2011) Ecological physiology of diet and digestive systems. Annu Rev Physiol 73(1):69-93

Kim DH, Brunt J, Austin B (2007) Microbial diversity of intestinal contents and mucus in rainbow trout (Oncorhynchus mykiss). J Appl Microbiol 102(6):1654-1664

Langille MG, Zaneveld J, Caporaso JG, McDonald D, Knights D, Reyes JA, Clemente JC, Burkepile DE, Thurber RL, Knight R, Beiko RG (2013) Predictive functional profiling of microbial communities using $16 \mathrm{~S}$ rRNA marker gene sequences. Nat Biotechnol 31(9):814-821

Li JN, Wei MF, Yu DP (2001) Separation and identification of a pathogenic bacterium in Fugu obscurus. Reserv Fish 5:42-43

Liu Z, Lozupone C, Hamady M, Bushman FD, Knight R (2007) Short pyrosequencing reads suffice for accurate microbial community analysis. Nucleic Acids Res 35(18):e120-e120

Loudon AH, Woodhams DC, Parfrey LW, Archer H, Knight R, Mckenzie V, Harris RN (2014) Microbial community dynamics and effect of environmental microbial reservoirs on red-backed salamanders (Plethodon cinereus). ISME J 8(4):830-840

Lozupone C, Hamady M, Knight R (2006) Unifrac_-an online tool for comparing microbial community diversity in a phylogenetic context. BMC Bioinform 7(1):371

Lu ZH, Li W, Shi P, Wang ZJ (2011) Study on composition and generation of predominant intestinal bacteria in cultured Percoypris pingi pingi. Freshw Fish 41(3):29-33

Lyons PP, Turnbull JF, Dawson KA, Crumlish M (2017) Phylogenetic and functional characterization of the distal intestinal microbiome of rainbow trout oncorhynchus mykiss from both farm and aquarium settings. J Appl Microbiol 122(2):347-363 
Mandal SC, Hasan M, Rahman MS, Manik MH, Mahmud ZH, Sirajul Islam MD (2009) Coliform bacteria in Nile Tilapia, Oreochromis niloticus of ShrimpGher, pond and fish market. World J Fish Mar Sci 1(3):160-166

Penn K, Jenkins C, Nett M, Udwary DW, Gontang EA, Mcglinchey RP, Foster B, Lapidus A, Podell S, Allen EE, Moore BS, Jensen PR (2009) Genomic islands link secondary metabolism to functional adaptation in marine actinobacteria. ISME J 3(10):1193-1203

Pérez T, Balcázar JL, Ruiz-Zarzuela I, Halaihel N, Vendrell D, de Blas I, Múzquiz JL (2010) Host-microbiota interactions within the fish intestinal ecosystem. Mucosal Immunol 3(4):355-360

Reveco FE, Øverland M, Romarheim OH, Mydland LT (2014) Intestinal bacterial community structure differs between healthy and inflamed intestines in Atlantic salmon (Salmo salar L.). Aquaculture 420(3):262-269

Ringø E, Olsen R, Mayhew TM, Myklebust R (2003) Electron microscopy of the intestinal microflora of fish. Aquaculture 227(1):395-415

Romero J, Navarrete P (2006) 16s rDNA-based analysis of dominant bacterial populations associated with early life stages of Coho salmon (Oncorhynchus kisutch). Microb Ecol 51(4):422-430

Round JL, Mazmanian SK (2009) The gut microbiota shapes intestinal immune responses during health and disease. Nat Rev Immunol 9:313-323

Schloss PD, Westcott SL, Ryabin T, Hall JR, Hartmann M, Hollister EB, Lesniewski RA, Oakley BB, Parks DH, Robinson CJ, Sahl JW, Stres B, Thallinger GG, Van Horn DJ, Weber CF (2009) Introducing mothur: open-source, platformindependent, community-supported software for describing and comparing microbial communitie. Appl Environ Microbiol 75(23):7537-7541

Strolnsky A, Schnelder Z (1987) Cobamide dependent enzymes. In: Schneider Z, Stroinsky A (eds) Comprehensive $B_{12}$. de Gruyter, Ber lin, pp 225-266

Tian YS, Chen ZF, Duan HM, Ma WH, Tang J, Li WS, Liu JC, Hou YX, Sun ZX, Pang ZF, Wang XM, Zhai JM, Li M (2017) The family line establishment of the

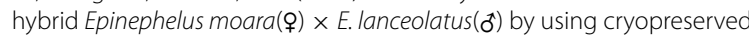
sperm and the related genetic effect analysis. J Fish China 41(10):1-12

Tsuchiya C, Sakata T, Sugita H (2008) Novel ecological niche of Cetobacterium somerae, an anaerobic bacterium in the intestinal tracts of freshwater fish. Lett Appl Microbiol 46(1):43-48

Wang C, Sun GX, Li SS, Li X, Liu Y (2018) Intestinal microbiota of healthy and unhealthy atlantic salmon Salmo salar $\mathrm{L}$. in a recirculating aquaculture system. Trans Oceanol Limnol 36(2):414-426

Willing BP, Russell SL, Finlay BB (2011) Shifting the balance: antibiotic effects on host-microbiota mutualism. Nat Rev Microbiol 9(4):233-243
Wu S, Wang G, Angert ER, Wang W, Li W, Zou H (2012) Composition, diversity, and origin of the bacterial community in grass carp intestine. PLoS ONE 7(2):e30440

Wu SG, Tian JY, Gatesoupe FJ, Li WX, Zou H, Yang BJ, Wang GT (2013) Intestinal microbiota of gibel carp (Carassius auratus gibelio) and its origin as revealed by 454 pyrosequencing. World J Microbiol Biotechnol 29(9):1585-1595

Wu J, Peters BA, Dominianni C, Zhang Y, Pei Z, Yang L, Ma Y, Purdue MP, Jacobs EJ, Gapstur SM, Li H, Alekseyenko AV, Hayes RB, Ahn J (2016a) Cigarette smoking and the oral microbiome in a large study of american adults. ISME J 10(10):2435-2446

Wu SQ, Zheng LY, Huang ZC, Chou DG, Xu CH, Zhen JP, Qiu FY, Wu JL, Liu YH (2016b) Embryonic and morphological development in larva, juvenile, and young stages of hybrid grouper (Epinephelus moara $\$ \times$ Elanceolatusơ). J Fish Res 38(1):27-35

Wu SW, Gao TH, Zheng YZ, Wang WW, Cheng YY, Wang GT (2016c) Microbial diversity of intestinal contents and mucus in yellow catfish (Pelteobagrus fulvidraco). Aquaculture 303(1):1-7

Xie DZ, Yang LP, Yu RM, Chen F, Lu RH, Qin CB, Nie GX (2017) Effects of dietary carbohydrate and lipid levels on growth and hepatic lipid deposition of juvenile tilapia, Oreochromis niloticus. Aquaculture 479:696-703

Xing DC, Song XF, Peng L, Cheng YW, Zhai JM (2017) Effects of temperature and salinity on oxygen consumption rate and ammonia excretion rate of junevile Epinephelus grouper. Fish Modernizzation 44(3):7-14

Yang G, Jian SQ, Cao HZ, Wen CG, Hu BQ, Peng M, Peng LS, Yuan JG, Liang LF (2018) Changes in microbiota along the intestine of grass carp (Ctenopharyngodon idella): community, interspecific interactions, and functions. Aquaculture 498:151-161

Zhang Z, Liao MJ, Li B, Wang YG, Wang L, Rong XJ, Chen GP (2014) Study on cultured half-smooth tongue sole(Cynoglossus semilaevis Günther) intestinal microflora changes affected by different disease occurrence. J Fish China 38(9):1565-1572

\section{Publisher's Note}

Springer Nature remains neutral with regard to jurisdictional claims in published maps and institutional affiliations.

\section{Submit your manuscript to a SpringerOpen ${ }^{\odot}$ journal and benefit from:}

- Convenient online submission

- Rigorous peer review

- Open access: articles freely available online

- High visibility within the field

Retaining the copyright to your article

Submit your next manuscript at $\boldsymbol{\nabla}$ springeropen.com 